\title{
Motivación Hacia Las Clases De Educación Física En Preadolescentes Mexicanos De Acuerdo Al Género
}

\author{
Perla Jannet Jurado García, (Dra.) \\ Ma. Concepción Soto Valenzuela (Dra.) \\ Juan Francisco Aguirre Chávez (Dr.) \\ Raúl Josué Nájera Longoria (Dr.) \\ Fernando Mondaca Fernández (M.C.) \\ Autonomous University of Chihuahua, Mexico
}

Doi:10.19044/esj.2018.v14n31p206 URL:http://dx.doi.org/10.19044/esj.2018.v14n31p206

\begin{abstract}
The aim of the present research was to compare the motivation towards physical education profiles from Mexican elementary school students. The total sample included 274 students (140 girls) from Chihuahua City, Mexico; children's ages ranged from 10 to 12 years. A quantitative approach with a descriptive transversal design was used. Results from a one-way MANOVA followed by univariate analyses of variance showed that compared to boys, girls report higher levels of intrinsic motivation $(\mathrm{F}=19.188, \mathrm{p}<.001)$, integrated regulation $(\mathrm{F}=13.730, \mathrm{p}<.001)$, identified regulation $(\mathrm{F}=11.902$, $\mathrm{p}<.001)$ and introjected regulation $(\mathrm{F}=6.937, \mathrm{p}<.01)$; no significant differences were encountered in external regulation and demotivation. These results indicate that, in general, women show a better profile of motivation towards physical education classes than men. However, it is necessary to develop more research because the issue transcends the scope of the present study.
\end{abstract}

Keywords: Locus of causality, gender, motivation, self-perceptions

\section{Resumen}

El objetivo de esta investigación consistió comparar los perfiles de motivación hacia las clases de educación física de estudiantes de educación básica mexicanos. La muestra total fue de 274 sujetos; 140 niñas y 134 niños, estudiantes de educación básica de la ciudad de Chihuahua México, con edades entre los 10 y 12 años. El abordaje adoptado en la investigación se enmarcó dentro de un enfoque cuantitativo con un diseño descriptivo tipo encuesta. Los resultados del análisis multivariante de la varianza, seguido por los análisis de varianza univariados, muestran que las niñas son las que reportan mayores 
niveles de motivación intrínseca $(\mathrm{F}=19.188, \mathrm{p}<.001)$, regulación integrada $(\mathrm{F}=13.730, \mathrm{p}<.001)$, regulación identificada $(\mathrm{F}=11.902, \mathrm{p}<.001) \mathrm{y}$ regulación introyectada $(\mathrm{F}=6.937, \mathrm{p}<.01)$; y sin diferencias significativas en regulación externa y desmotivación. Estos resultados revelan que en general las mujeres en comparación con los hombres muestran un mejor perfil de motivación hacia las clases de educación física. No obstante, es preciso desarrollar más investigación al respecto pues el tema trasciende del todo los alcances de la presente investigación.

Palabras Clave: Locus de causalidad, género, motivación, autopercepciones

\section{Introducción:}

La clase de educación física ofrece oportunidades para mejorar las relaciones sociales, la cooperación y el nivel de responsabilidad (Baena, RuizJuan, \& Granero-Gallegos, 2016), además promueve el compromiso de los adolescentes y proporciona soporte a la práctica de actividad física y deportiva en el tiempo libre (Jaakkola, Washington, \& Yli-Piipari, 2013);

Entre los motivos más importante para la realización de actividad física se encuentra la prevención y la salud positiva, por ello, se debe proveer de un contexto estructurado que promueva la autonomía del alumnado, estrategias para favorecer la diversión durante la clase lo que parece tener un efecto positivo sobre la generación de patrones motivacionales adaptativos (Franco, Coterón, Gómez, Brito, \& Martínez, 2017).

Así pues, la motivación en las clases de educación física ha sido un foco de estudio y preocupación por parte de los investigadores (Teixeira, Monteiro, Carraça, \& Palmeira, 2018); y uno de principales marcos utilizados para estudiar los procesos de la motivación ha sido la teoría de la autodeterminación; la cual se derivada empíricamente de la motivación humana y la personalidad en contextos sociales que diferencia la motivación en términos de ser autónomo y controlado. Esta teoría postula dos tendencias innatas de crecimiento para explicar la vitalidad, el desarrollo y la adaptación psicológica de las personas, a saber, la motivación e internalización intrínsecas; donde la motivación intrínseca se refiere a la energía innata que las personas manifiestan cuando persiguen una meta o una actividad porque es interesante $\mathrm{o}$ divertida. La distinción entre motivación intrínseca $\mathrm{e}$ internalización gira en torno a la forma en que un individuo se siente atraído por participar en una actividad determinada (Deci \& Ryan, 2012).

La teoría de la autodeterminación pues, proporciona un marco útil para comprender la motivación de los niños y adolescentes para actividad física; además, esta teoría se asocia con un comportamiento sostenido de promoción de la salud (Mayorga-Vega \& Viciana, 2014; Owen, Smith, Lubans, Ng, \& Lonsdale, 2014); de ahí la importancia de comprender los factores que afectan 
la motivación de las personas para la práctica de actividad física, dado que puede tener un impacto a largo plazo.

Se han desarrollado diversos instrumentos para medir las percepciones de los estudiantes sobre su motivación, entre los que se encuentra el locus de causalidad percibido, dicho instrumento se ha empleado para examinar las regulaciones motivacionales de los estudiantes hacia la educación física a nivel contextual (Lonsdale, Sabiston, Taylor, \& Ntoumanis, 2011; Teixeira et al., 2018).

Por otro lado, se afirma que existen diferencias en relación al tipo de motivación autodeterminada de acuerdo al género (Amado, Sánchez-Miguel, Leo, Sánchez-Oliva, \& García-Calvo, 2014); además, las motivaciones que las mujeres manifiestan en la práctica de actividad física y deportiva son diferentes a las del género masculino (Grao-Cruce, Fernández-Martínez, Teva-Villén \& Niviala, 2017).

En este estudio se intenta determinar las diferencias y similitudes entre niñas y niños de educación básica mexicanos en cuanto a sus perfiles de motivación hacia las clases de educación física. En consecuencia, esta investigación pretende, como investigación aplicada, aportar información que se traduzca en una práctica educativa de mayor calidad en el contexto de atención a la diversidad; contribuyendo al saber pedagógico en el esclarecimiento de los factores que conforman un modelo de desarrollo humano integral; bajo la premisa de que los esfuerzos educativos deben enfocarse hacia el aumento de los sentimientos de autovaloración y competencia de los estudiantes, fortaleciendo la autoestima y el autoconcepto, lo que a su vez favorecerá la motivación hacia el logro, las relaciones interpersonales y en general la forma particular de desenvolverse frente a diversas tareas y desafíos que se les presenten.

\section{Método}

\section{Participantes}

Participaron en el estudio 274 sujetos, 140 niñas y 134 niños estudiantes de educación básica de la ciudad de Chihuahua, México. La edad de los sujetos fluctuó entre los 10 y 12 años, con $\mathrm{M}=10.72 \pm 0.75$ años para las niñas y $\mathrm{M}$ $=10.62 \pm 0.65$ años para los niños.

La muestra se obtuvo mediante un muestreo por conveniencia, tratando de abarcar la representatividad para alumnos que cursan el quinto y sexto de primaria en la Ciudad de Chihuahua, México.

\section{Instrumento}

Para medir los perfiles de motivación hacia las clases de educación física se utilizó la Escala de Locus Percibido de Causalidad con la regulación integrada (PLOC-2) adaptada por Ferriz, González-Cutre, y Sicilia (2015) la 
cual consta de 6 factores con 4 ítems cada uno, que miden motivación intrínseca (ítems 1, 7, 13 y 19, porque la educación física es divertida; porque disfruto aprendiendo nuevas habilidades; porque la educación física es estimulante; $y$, por la satisfacción que siento mientras aprendo nuevas habilidades/técnicas), regulación integrada (ítems 2, 8, 14, 20, porque está de acuerdo con mi forma de vida; porque considero que la educación física forma parte de mí; porque veo la educación física como una parte fundamental de lo que soy; y, porque considero que la educación física está de acuerdo con mis valores), regulación identificada $(3,9,15,21$, porque quiero aprender habilidades deportivas; porque es importante para mí hacerlo bien en educación física; porque quiero mejorar en el deporte; $\mathrm{y}$, porque puedo aprender habilidades que podría usar en otras áreas de mi vida), regulación introyectada (ítems $4,10,16,22$, porque quiero que el/la profesor/a piense que soy un/a buen/a estudiante; porque me sentiría mal conmigo mismo si no lo hiciera; porque quiero que los/as otros/as estudiantes piensen que soy hábil; $\mathrm{y}$, porque me preocupa cuando no lo hago), regulación externa (ítems 5, 11, 17 y 23 , porque tengo problemas si no lo hago; porque eso es lo que se supone que debo hacer; para que el/la profesor/a no me grite; y, porque esa es la norma); y desmotivación (ítems $6,12,18,24$, pero no sé realmente por qué; pero no comprendo por qué debemos tener educación física; pero realmente siento que estoy perdiendo mi tiempo en educación física; $y$, pero no puedo comprender lo que estoy sacando de la educación física). El instrumento utiliza una escala tipo Likert de 1 (totalmente en desacuerdo) a 7 (totalmente de acuerdo).

\section{Diseño}

En cuanto al diseño del estudio, se utilizó un enfoque cuantitativo con un diseño descriptivo y transversal tipo encuesta (Hernández, Fernández, \& Baptista, 2014). La variable independiente fue el género y las variables dependientes el promedio de las puntuaciones en los factores motivación intrínseca, regulación integrada, regulación identificada, regulación introyectada, regulación externa y desmotivación del cuestionario Locus Percibido de Causalidad.

\section{Procedimiento}

Una vez conseguido el permiso tanto de las autoridades educativas como el de las familias, se invitó a participar en el estudio a niños y niñas estudiantes de la ciudad de Chihuahua, México. Los que aceptaron participar firmaron el consentimiento informado. Luego se aplicó el instrumento antes descrito en una sesión de aproximadamente 30 minutos; en las aulas de los centros educativos. Al inicio de la sesión se explicó cómo acceder al instrumento. Se solicitó la máxima sinceridad y se garantizó la confidencialidad de los datos que se obtuvieran. Las instrucciones se encontraban en las primeras pantallas 
de la versión computarizada del instrumento. Al término de la sesión se les agradeció su participación. Una vez aplicado el instrumento se recopilaron los resultados por medio del módulo generador del editor de escalas versión 2.0 (Blanco et al., 2013), y finalmente se analizaron mediante el paquete SPSS 18.0 .

\section{Análisis de datos}

Se realizaron análisis estadísticos descriptivos (medias y desviaciones estándar) para todas las variables dependientes. Posteriormente, después de verificar que los datos cumplían con los supuestos de los análisis estadísticos paramétricos, se llevó a cabo un análisis multivariante de varianza (MANOVA), seguido por los análisis de varianza univariados (ANOVAs), para examinar las diferencias entre niñas y niños en cuanto a su locus percibido de causalidad, el tamaño del efecto se estimó mediante el eta cuadrado $\left(\eta^{2}\right)$. Todos los análisis estadísticos se realizaron con el programa SPSS versión 18.0 para Windows. El nivel de significación estadística se estableció en $p=.05$.

\section{Resultados}

La Tabla 1 muestra los valores de las medias y las desviaciones estándar de las puntuaciones en los factores de Locus Percibido de Causalidad, así como los resultados del MANOVA y los subsiguientes ANOVAs. Los resultados del MANOVA mostraron diferencias globales estadísticamente significativas de acuerdo a la variable género en los puntajes promedio de los factores de Locus Percibido de Causalidad (Wilks' $\lambda=.929 ; p<.01 ; \eta^{2}=.071$ ). Posteriormente, los resultados de los ANOVAs mostraron que las niñas reportan mayores niveles de motivación intrínseca $(F=19.188, p<.001)$, regulación integrada $(F=13.730, p<.001)$, regulación identificada $(F=11.902, p<.001) \mathrm{y}$ regulación introyectada $(F=6.937, p<.01)$; y sin diferencias significativas en regulación externa y desmotivación.

Tabla 1. Resultados MANOVA y ANOVAs para los puntajes promedio de los factores de Locus Percibido de Causalidad de acuerdo al género.

\begin{tabular}{lccccc}
\hline & $\begin{array}{c}\text { hombres } \\
(n=134)\end{array}$ & $\begin{array}{c}\text { mujeres } \\
(n=140)\end{array}$ & $F$ & $p$ & $\eta^{2}$ \\
\hline & & & 3.380 & $<.01$ & .071 \\
motivación intrínseca & $3.98(0.84)$ & $4.35(0.55)$ & 19.188 & $<.001$ & .066 \\
regulación integrada & $3.84(0.86)$ & $4.20(0.80)$ & 13.730 & $<.001$ & .048 \\
regulación identificada & $4.07(0.74)$ & $4.36(0.63)$ & 11.902 & $<.001$ & .042 \\
regulación introyectada & $3.36(0.89)$ & $3.65(0.92)$ & 6.937 & $<.001$ & .025 \\
regulación externa & $3.15(0.98)$ & $3.28(0.99)$ & 1.170 & $>.05$ & \\
desmotivación & $2.52(1.12)$ & $2.59(1.14)$ & 0.230 & $>.05$ & \\
\hline
\end{tabular}

Nota. Los valores descriptivos se presentan como media (desviación estándar) 


\section{Conclusión:}

De los resultados mostrados y de su análisis, se pueden obtener las siguientes conclusiones:

1. Los resultados revelan que en general las mujeres en comparación con los hombres muestran un mejor perfil de motivación hacia las clases de educación física.

2. Las diferencias encontradas entre niñas y niños con respecto a su motivación en las clases de educación física sugieren además que al diseñar cualquier tipo de intervención que tenga como potenciar dicha motivación habrá que tomar en cuenta a la variable género; no obstante, es preciso desarrollar más investigación al respecto pues el tema trasciende del todo los alcances de la presente investigación. Se subraya además la importancia de realizar un mayor número de investigaciones sobre el tema en nuestro país.

Por último, cuando menos dos limitaciones están presentes en este trabajo. La primera es que los participantes son solo estudiantes entre 10 y 12 años, lo que supone una amenaza para la posibilidad de generalizar estos resultados. Ampliar la muestra (agregando por ejemplo estudiantes de otras edades) es un área de trabajo de cara al futuro. La segunda limitación proviene del propio instrumento de medición, que se basa en el autoinforme y que por ello puede contener los sesgos que se derivan de la deseabilidad social.

\section{References:}

1. Amado, D., Sánchez-Miguel, P. A., Leo, F. M., Sánchez-Oliva, D., \& García-Calvo, T. (2014). Diferencias de género en la motivación y percepción de utilidad del deporte escolar. Revista internacional de Medicina y Ciencias de la Actividad Física del Deporte, 14(56), 651664.

2. Baena, A., Ruiz-Juan, F., \& Granero-Gallegos, A. (2016). A crosscultural analysis in predicting $2 \times 2$ achievement goals in physical education based on social goals, perceived locus of causality and causal attribution. Studia Psychologica, 58(1), 74.

3. Blanco, H., Ornelas, M., Tristán, J. L., Cocca, A., Mayorga-Vega, D., López-Walle, J., \& Viciana, J. (2013). Editor for creating and applying computerise surveys. Procedia Social and Behavioral Sciences, 106, 935-940. doi:10.1016/j.sbspro.2013.12.105

4. Deci, E. L., \& Ryan, R. M. (2012). Self-Determination Theory. En P. A. M. Van Lange, A. W. Kruglanski, \& E. T. Higgins (Eds.), Handbook of Theories of Social Psychology: Collection: Volumes $1 \& 2$ (pp. $416-$ 437): SAGE Publications.

5. Ferriz, R., González-Cutre, D., \& Sicilia, Á. (2015). Revisión de la Escala del Locus Percibido de Causalidad (PLOC) para la inclusión de 
la medida de la regulación integrada en educación física. Revista de psicología del deporte, 24(2), 329-338.

6. Franco, E., Coterón, J., Gómez, V., Brito, J., \& Martínez, H. A. (2017). Influencia de la motivación y del flow disposicional sobre la intención de realizar actividad físico-deportiva en adolescentes de cuatro países. RETOS. Nuevas tendencias en Educación Física, Deporte $y$ Recreación, (31).

7. Grao-Cruce, A., Fernández-Martínez, A., Teva-Villén, M. R., \& Niviala, A. (2017). Autoconcepto físico e intencionalidad para ser físicamente activo en los participantes del programa de escuelas deportivas. Journal of Sport and Health Research, 9(1), 15-26.

8. Hernández, R., Fernández, C., \& Baptista, P. (2014). Metodología de la investigación. México: McGraw- Hill.

9. Jaakkola, T., Washington, T., \& Yli-Piipari, S. (2013). The association between motivation in school physical education and self-reported physical activity during Finnish junior high school: A selfdetermination theory approach. European Physical Education Review, 19(1), 127-141. doi:10.1177/1356336X12465514

10. Lonsdale, C., Sabiston, C. M., Taylor, I. M., \& Ntoumanis, N. (2011). Measuring student motivation for physical education: examining the psychometric properties of the perceived locus of causality questionnaire and the situational motivation scale. Psychology of Sport and Exercise, 12(3), 284-292. doi:10.1016/j.psychsport.2010.11.003

11. Mayorga-Vega, D., \& Viciana, J. (2014). Adolescents' physical activity in physical education, school recess, and extra-curricular sport by motivational profiles. Perceptual and motor skills, 118(3), 663-679. doi:10.2466/06.10.PMS.118k26w0

12. Owen, K. B., Smith, J., Lubans, D. R., Ng, J. Y., \& Lonsdale, C. (2014). Self-determined motivation and physical activity in children and adolescents: A systematic review and meta-analysis. Preventive medicine, 67, 270-279. doi:10.1016/j.ypmed.2014.07.033

13. Teixeira, D. S., Monteiro, D., Carraça, E., \& Palmeira, A. L. (2018). Translation and validation of the perceived locus of causality questionnaire (PLOCQ) in a sample of portuguese physical education students. Motriz: Revista de Educação Física, 24(2). doi:10.1590/s1980-6574201800020007 